\title{
PAPER
}

\section{Geometrically self-similar ion acceleration in collisionless plasma beam expansion}

To cite this article: Yuan Hu et al 2020 Plasma Sources Sci. Technol. 29125004

View the article online for updates and enhancements.

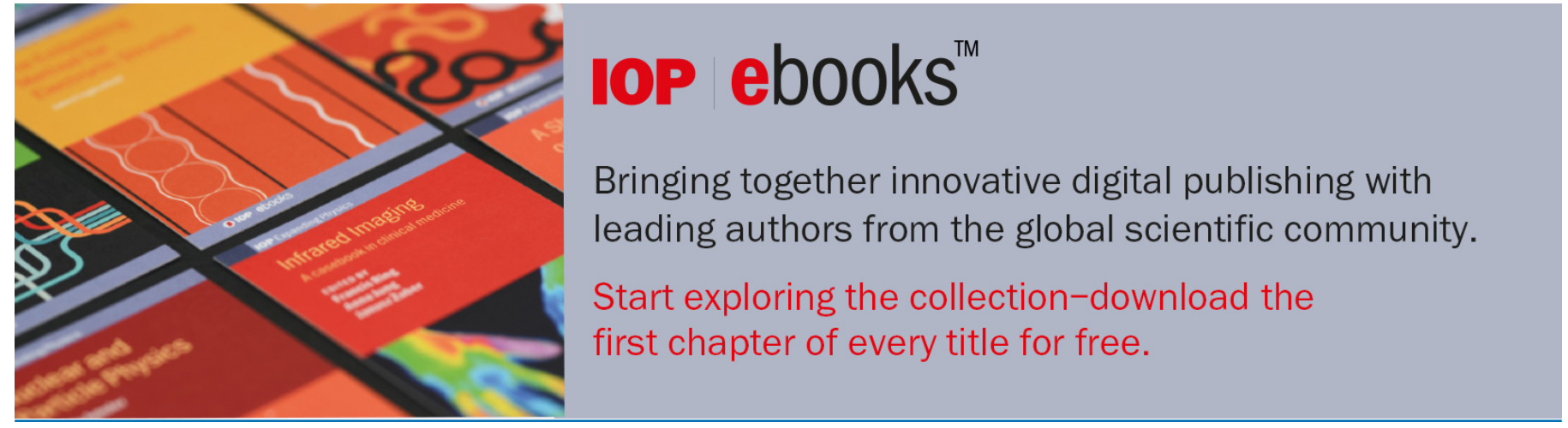

This content was downloaded from IP address 159.226 .231 .70 on 13/01/2021 at 06:41 


\title{
Geometrically self-similar ion acceleration in collisionless plasma beam expansion
}

\author{
Yuan $\mathrm{Hu}^{1}{ }^{\oplus}$, Joseph Wang ${ }^{2} \odot$ and Quanhua Sun ${ }^{1,3, *}$ \\ 1 State Key Laboratory of High Temperature Gas Dynamics, Institute of Mechanics, Chinese Academy of \\ Sciences, Beijing 100190, People's Republic of China \\ 2 Department of Astronautical Engineering, University of Southern California, Los Angeles, CA 90089, \\ United States of America \\ 3 School of Engineering Science, University of Chinese Academy of Sciences, Beijing 100049, People's \\ Republic of China
}

E-mail: yhu@imech.ac.cn, josephjw@usc.edu and qsun@imech.ac.cn

Received 6 June 2020, revised 29 September 2020

Accepted for publication 8 October 2020

Published 9 December 2020

\begin{abstract}
Fully kinetic particle-in-cell simulations are carried out to study ion acceleration in a geometrically expanding plasma beam. The ion acceleration is demonstrated to be essentially self-similar. An analytical model is developed for ion acceleration evaluation. The plasma plume is found to be fully expanded after about 20 times of the beam radius $\left(R_{0}\right)$ downstream of the beam exit, where the ion velocity reaches the maximum. The maximum ion velocity increase is about twice of the initial ion acoustic speed. Hence, to minimize the facility effects on plasma plume experiments in a vacuum chamber, the chamber needs to provide a plume expansion region of at least $20 R_{0}$ along the beam direction so the expansion process is not terminated prematurely.
\end{abstract}

Keywords: geometrically expanding plasma, self-similarity, ion acceleration, facility effects, fully kinetic

(Some figures may appear in colour only in the online journal)

\section{Introduction}

Plasma sources are widely used to generate low temperature, rarefied plasma beams for applications in spacecraft charging control, electric propulsion (EP), and laboratory simulations of space plasmas. In such applications, the relevant characteristic length of interest is often much smaller than the collision mean free path in the beam, and thus the plasma beams can be considered collisionless downstream of the exit. Depending on the application, the emitting velocity of the ions in the beam can range from very low (e.g. plasma contactors) to very high (e.g. EP thrusters). Depending on the strength of the static magnetic field applied to the plasma source, the beam expansion may be geometrically or magnetically guided. However, the dominating mechanism affecting the ion dynamics in the

* Author to whom any correspondence should be addressed. beam is similar, i.e. the conversion of electron thermal energy into ion beam energy.

The expansion of a collisionless plasma beam is a classical subject in plasma dynamics as well as a practical problem related to plasma source design and performance. Most previous studies on plasma beam expansion were carried out within the context of either one-dimensional (1D) plasma expansion [1-4] (and references therein) or EP thruster plumes [5-10] (and references therein). However, one important question that has not been addressed is whether the geometric self-similarity exists in an expanding collisionless plasma beam. The geometric self-similarity is not only an important physics question but also has practical implications. For instance, plasma source design involves testing of prototype models at different sizes; laboratory testing of plasma sources needs to consider the boundary effects of a vacuum chamber on plasma beam expansion [11-13]; and computer simulations are often 


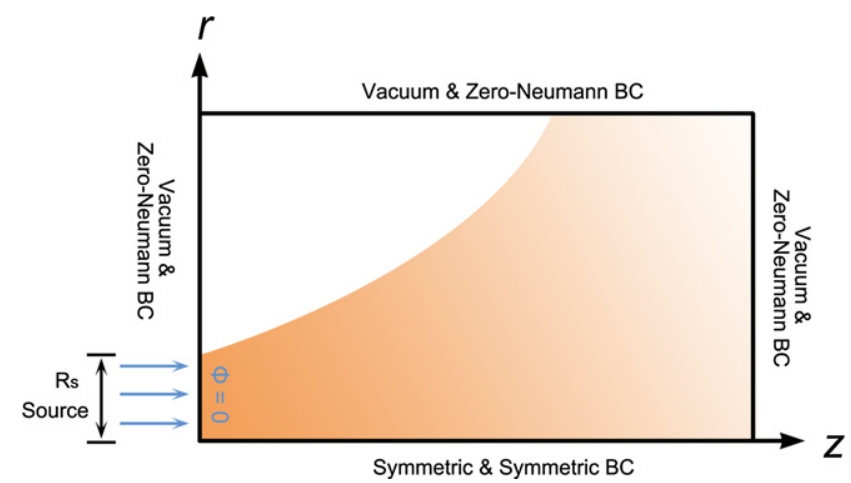

Figure 1. Schematic of simulation setup.

performed at scale-down size due to computational limitations $[14,15]$. The validity of self-similarity determines whether a specific set of results may be applied to beams emitted from similar sources of different sizes or from the same source but under different operating conditions or experimental settings.

This paper addresses whether ion acceleration is geometrically self-similar in collisionless plasma beam expansion. Previous experiments on a geometrically expanding plasma beam showed that ion beam energy increases with decreasing source radius $[16,17]$, suggesting that the ion acceleration depends on the plasma source size, and thus the geometric self-similarity may not be satisfied. Few theoretical or modeling studies have been carried out to evaluate the ion acceleration vs different beam sizes. Here, we carry out axi-symmetric fully kinetic particle-in-cell (PIC) simulations of geometrically expanding collisionless plasma beams of different beam radii, and derive an analytical model for ion acceleration evaluation. Section 2 discusses the simulation model and setup. Section 3 first addresses the self-similarity in ion acceleration and then discusses the effects from electron cooling and collisions on self-similarity. Section 4 contains a summary and conclusions.

\section{Simulation model}

Figure 1 shows the schematic of the problem setup. We consider that a plasma beam is injected into a vacuum from a source. The beam has an initial radius of $R_{0}$, and the emission surface is located at $(z, r)=\left(0,0 \sim R_{0}\right)$. This setup considers a beam expansion process similar to the experiments of references $[16,17]$, except that the expansion here occurs in an unbounded space.

The simulations are carried out using an axi-symmetric fully kinetic electrostatic PIC code, in which both the ions and electrons are treated as particles. The particle position $\mathbf{x}$ and velocity $\mathbf{v}$, the ion number density $n_{\mathrm{i}}$ and electron number density $n_{\mathrm{e}}$, and the electric potential $\Phi$ are solved self-consistently from Newton's equation of motion and Poisson's equation in a cylindrical coordinate system

$$
\mathbf{v}=\frac{\mathrm{d} \mathbf{x}}{\mathrm{d} t}, \quad \frac{\mathrm{d}}{\mathrm{d} t}(m \mathbf{v})=\mathbf{F}, \quad \mathbf{F}=-q \nabla \Phi
$$

$$
-\nabla^{2} \Phi=\frac{e}{\epsilon_{0}}\left(n_{\mathrm{i}}-n_{\mathrm{e}}\right)
$$

where $q= \pm e$ and $m$ denote particle charge and mass, respectively, $e$ the elementary charge, and $\epsilon_{0}$ the vacuum permittivity. PIC simulations are carried out using the proton to electron mass ratio, $m_{\mathrm{i}} / m_{\mathrm{e}}=1836$. This allows us to account for the full electron kinetic effects during the expansion while keeping the computational cost of the fully kinetic PIC simulations acceptable [5, 18].

For a plasma source, the beam immediately outside of the emitting surface is affected mostly by the specifics of source design, which determines the sheath covering the exit surface and the initial ion-electron mixing process. The concept of self-similarity applies only to the region where the beam is neutralized and the potential of the emitting surface is shielded by the sheath. It is found that a well neutralized beam can form within a few Debye length from the exhaust plane for a wide variety of plasma sources [16, 17, 19-22]. Depending on the application and the operating condition, the ratio of the initial beam radius to the Debye length at the source exit typically ranges from tens to hundreds or thousands. Hence, the thickness of the sheath at the exit surface is negligible compared to the beam size, and the region to which the self-similarity applies is typically for $z / R_{0} \gtrsim O\left(10^{-2}\right) \sim$ $O\left(10^{-1}\right)$. The simulation model considers a quasi-neutral plasma beam downstream of the sheath. We use a virtual plasma source which emits both the electrons and ions from the same exit surface at $z=0$ to form a quasi-neutral and currentfree $\left(\mathbf{J}_{\mathrm{i}}+\mathbf{J}_{\mathrm{e}}=0\right)$ plasma beam. The potential of the emitting surface is fixed to be zero so that no effects from the source exit surface potential or sheath are included.

At each simulation step, macro-particles representing the ions and electrons are generated at the source exit surface and injected into the simulation domain as drifting Maxwellian distributions with the same beam velocity $v_{0}$ along the $z$ direction. The number density and temperature for the ions and electrons are chosen to be: $\tilde{n}_{\mathrm{e}}=n_{\mathrm{e}} / n_{0}=1, \tilde{n}_{\mathrm{i}}=n_{\mathrm{i}} / n_{0}=$ $1, \tilde{T}_{\mathrm{e}}=T_{\mathrm{e}} / T_{0}=1, \tilde{T}_{\mathrm{i}}=T_{\mathrm{i}} / T_{0}=0.025$. The beam velocity $\left(v_{0}\right)$ is chosen such that the Mach number $M=v_{0} / C_{s 0}=$ $v_{0} / \sqrt{k_{\mathrm{b}} T_{0} / m_{\mathrm{i}}}=1$, where $k_{\mathrm{b}}$ denotes the Boltzmann constant. This corresponds to $\tilde{v}_{0}=v_{0} / v_{\mathrm{te} 0}=v_{0} / \sqrt{k_{\mathrm{b}} T_{0} / m_{\mathrm{e}}} \simeq 0.0233$. The beam is assumed to have no initial divergence caused by the source design.

The lower boundary $(r=0)$ is the symmetric axis. All other boundaries are treated as an open boundary for particles. The zero-Neumann condition for the electric potential field is applied at all domain boundaries. The boundary condition for particles at the source emission surface and open boundaries need to be carefully handled to guarantee a quasineutral plasma in the simulation domain [19-21, 23]. At the open boundaries, the method presented in $\mathrm{Li}$ et al [7] is adopted and modified to maintain the current free condition. This method removes any ions reaching the domain boundary and reflects any escaping electrons with kinetic energy below a threshold back to the simulation domain. The reflection models the attraction of electrons back to the beam by the potential drop due to beam expansion. At the source emission 
surface, any backflow electrons are deleted from the domain. The reason is as follows. Once inside the source where the density is high and the collision is frequent, a backflow electron will become re-equilibrated and lose all its memory when it is re-injected into the beam at a later time. At steady state, these electrons are part of the electrons population emitted from the source. The potential will be self-adjusted to maintain the current-free and quasi-neutral conditions, which will be demonstrated later.

The mesh resolution used is $\Delta z=\Delta r=\lambda_{\mathrm{D} 0}$ and the simulation time step is $\Delta t=0.1 \omega_{\mathrm{pe} 0}^{-1}$, where $\lambda_{\mathrm{D} 0}=\sqrt{\epsilon_{0} k_{\mathrm{b}} T_{0} / n_{0} e^{2}}$ and $\omega_{\mathrm{pe} 0}=\sqrt{n_{0} e^{2} / \epsilon_{0} m_{\mathrm{e}}}$ are the Debye length and electron plasma frequency computed according to source plasma parameters, respectively. Note that both $\lambda_{\mathrm{D} 0}$ and $\omega_{\mathrm{pe} 0}^{-1}$ are the minimum values of the Debye length and electron plasma time scale, respectively, for the expansion plasma flow. Hence, the mesh resolution and time step used are well within the criteria to avoid artificial numerical heating and numerical instability in PIC for the entire simulation domain [24].

For quasi-neutral plasma beams, the expansion process is qualitatively similar if the ratio of the initial beam radius to the Debye length at the source exit $R_{0} / \lambda_{\mathrm{D} 0}>1$ [19]. The quantitative effects from the initial beam size on geometrical expansion is expected to be more significant for smaller $R_{0} / \lambda_{\mathrm{D} 0}$. In this paper, we carry out PIC simulations for a range of initial beam radius, for $R_{0} / \lambda_{\mathrm{D} 0}=2,5,8,10,15,20,30$ and 50. (Note that previous PIC simulations of collisionless plasma beam expansion were mostly limited to $R_{0} / \lambda_{\mathrm{D} 0} \leqslant 15$ [7, 25, 26].) For all the $R_{0} / \lambda_{\mathrm{D} 0} \leqslant 15$ cases, the size of the simulation domain $\left(z / \lambda_{\mathrm{D} 0}, r / \lambda_{\mathrm{D} 0}\right)$ used is $(0 \sim 2048$, $0 \sim 2048)$. For all the $R_{0} / \lambda_{\mathrm{D} 0} \geqslant 20$ cases, the size of the simulation domain used is $(0 \sim 3072,0 \sim 3072)$. A uniform particle weight is used to avoid the errors which will be introduced by particle splitting and merging in a non-uniform particle weighting scheme. The computational cost increases dramatically with the increase of beam size. The total number of macro-particles at the completion of a simulation run is about 50 million for $R_{0}=2 \lambda_{\mathrm{D} 0}$ while this number increases to 1.1 billion for $R_{0}=50 \lambda_{\mathrm{D} 0}$.

To obtain a steady state beam, each simulation needs to run for several ion transient periods $\left(t_{\text {itran }}\right)$ in the beam region of interest. Figure 2 compares 1D plasma density and ion velocity increment profile along the beam center line obtained at several different simulation times for the $R_{0} / \lambda_{\mathrm{D} 0}=10$ case. The results obtained at $t \omega_{\mathrm{pi}} \gtrsim 200$ are indistinguishable, indicating that a steady state is achieved at $t \omega_{\mathrm{pi}} \gtrsim 200$. In the simulation, the time it takes beam ion to travel from $z=0$ to $z=20 R_{0}$ is about $t \omega_{\mathrm{p} i 0} \simeq 7.5\left(R / \lambda_{\mathrm{D} 0}\right)$. Thus, for the $R_{0} / \lambda_{\mathrm{D} 0}=10 \mathrm{case}$, $t \omega_{\mathrm{pi}} \simeq 200$ corresponds to about 2.7 ion transient periods over a distance of $z / R_{0} \leqslant 20$ in the beam. However, to ensure the steady state, we aggressively run simulations $\geqslant 4.5 \times t_{\text {itran }}$ for the $R_{0} / \lambda_{\mathrm{D} 0} \leqslant 10$ cases while $\geqslant 3.5 \times t_{\text {itran }}$ for the $R_{0} / \lambda_{\mathrm{D} 0} \geqslant$ 15 cases due to computational constraint.

Finally, figure 3 shows the net electron and ion current (contribution from both inflow and backflow) passing through the source exit surface at the steady state. The current is normalized by $I_{0}=e n_{0} v_{0}\left(\pi R_{0}^{2}\right)$. Figure 3 confirms that the plasma beam emitted is current-free, $I_{\mathrm{i}}+I_{\mathrm{e}} \simeq 0$.
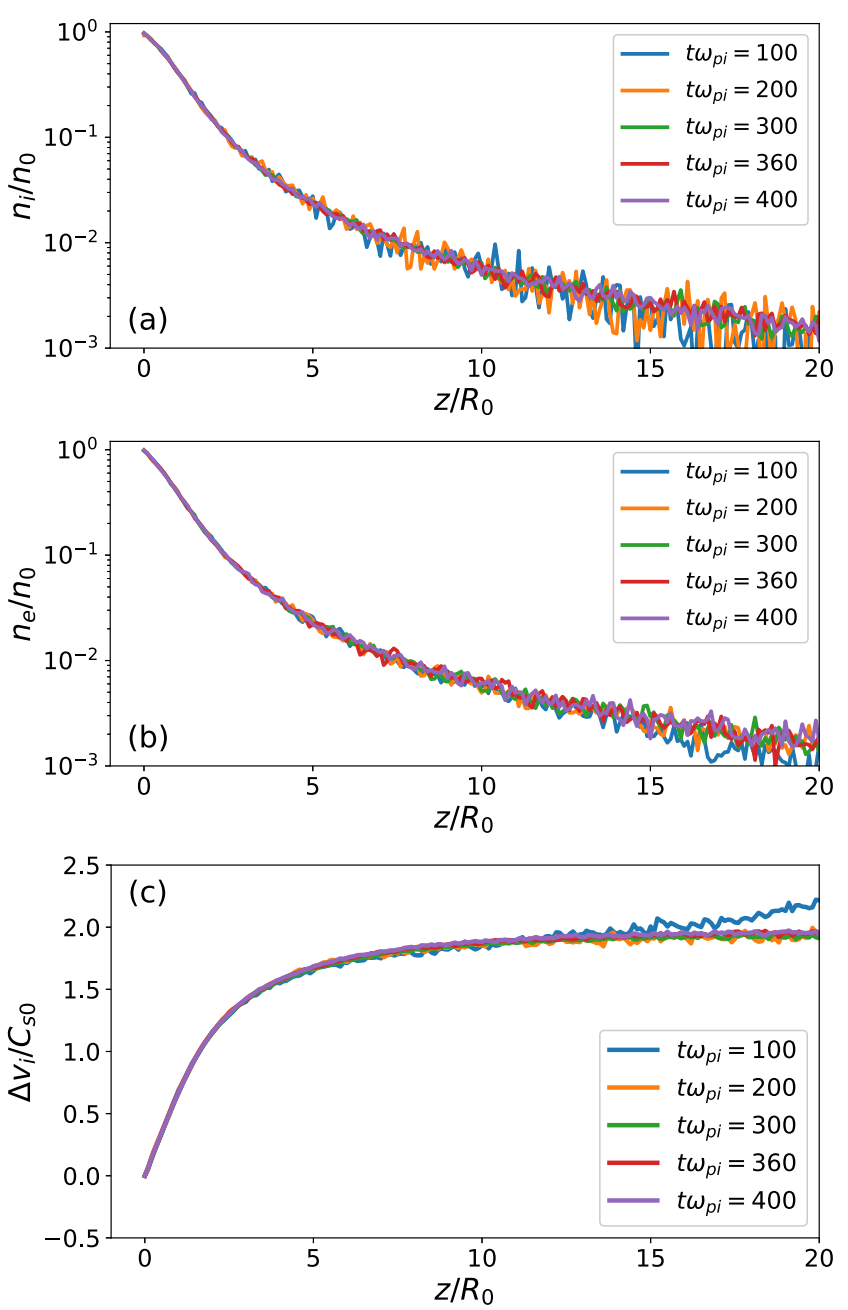

Figure 2. Comparison of the 1-D profiles along the beam center line at different simulation time steps for the $R_{0} / \lambda_{\mathrm{D} 0}=10$ case: (a) ion number density, (b) electron number density, and (c) ion acceleration.

\section{Results and discussions}

\subsection{PIC simulation results}

Corr et al [16] reported that the physics of a geometrically expanding plasma was affected considerably by the source size. For instance, the ion beam saw larger density and potential gradients, and achieved a higher beam energy as the radius of plasma source decreasing. Hence, we first carry out PIC simulations to compare with the experiments in [16]. Figure 4 shows the structure of a fully expanded plasma beam for the $R_{0} / \lambda_{\mathrm{D} 0}=10$ case. Note that, in [16], the beam radius at exit is about $R_{0} / \lambda_{\mathrm{D} 0}>300$ based on the parameter at the source/diffusion chamber interface. In order to compare the results in [16] with that in the simulation, figure 5 shows the 1D ion density profile along the beam axis. The density is normalized by $n_{0}$. To examine the self-similarity, the axial position is normalized by the initial beam radius, $z / R_{0}$. Figure 5 includes all the initial beam sizes considered: $R_{0} / \lambda_{\mathrm{D} 0}=2,5,8,10$, $15,20,30$ and 50, as well as the experimental data from [16]. To ensure the steady state achieved, the results shown for the 


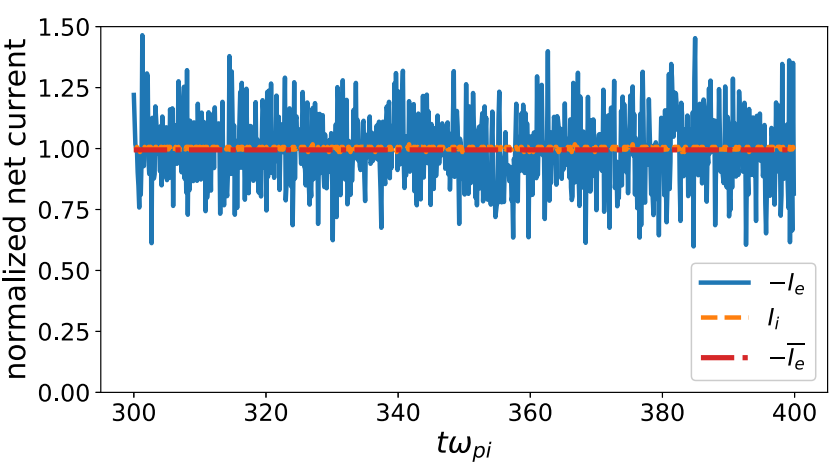

Figure 3. The current passing through the source exit plane for the $R_{0} / \lambda_{\mathrm{D} 0}=10$ case. $I_{\mathrm{e}}$ and $I_{\mathrm{i}}$ are the electron and ion current counted at every PIC time step, respectively. $\overline{I_{\mathrm{e}}}$ is $I_{\mathrm{e}}$ averaged over ever 100 $t \omega_{\mathrm{pi} 0}$.

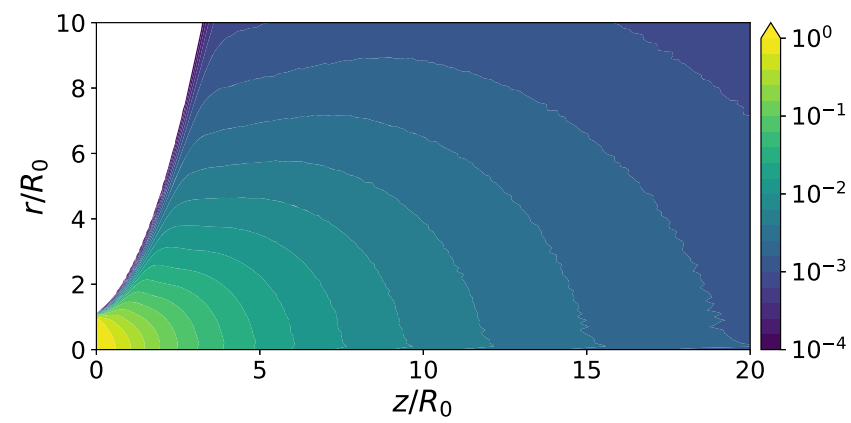

Figure 4. Ion number density contour for the $R_{0} / \lambda_{\mathrm{D} 0}=10$ case.

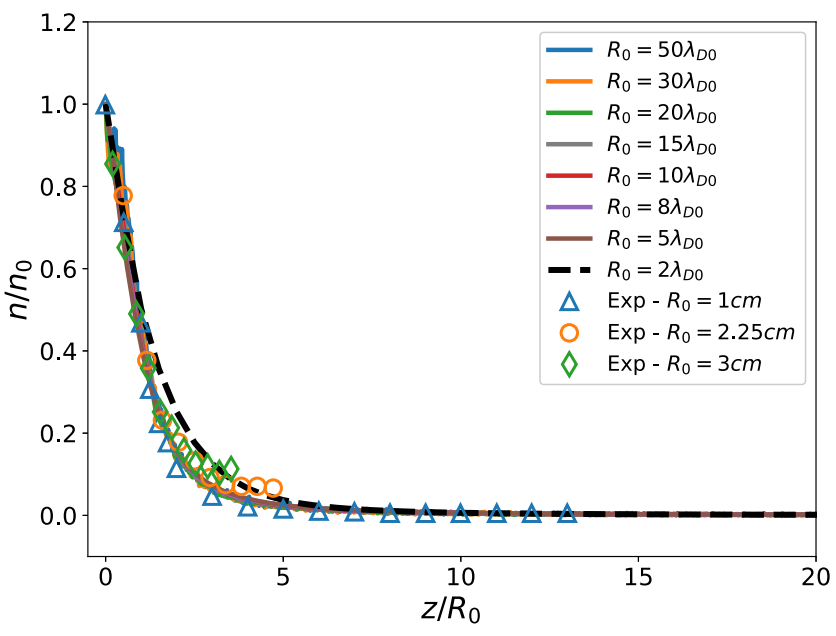

Figure 5. Ion number density along the beam axis: comparison of all PIC simulation cases with the experiment data (symbols) from [16].

$R_{0} / \lambda_{\mathrm{D} 0} \geqslant 15$ cases are limited to the region of $z / R_{0} \leqslant 10$ due to computational constraint.

Figure 5 shows that, except for the $R_{0} / \lambda_{\mathrm{D} 0}=2$ case, the density profiles from the PIC simulations are almost identical when the axial position is normalized as $z / R_{0}$. There is also an excellent agreement between the PIC results for the $R_{0} \geqslant 5 \lambda_{\mathrm{D} 0}$ cases and the experimental data. The density profile for the $R_{0} / \lambda_{\mathrm{D} 0}=2$ case from PIC shows a somewhat smaller gradient compared to other cases. For $R_{0} / \lambda_{\mathrm{D} 0}=2$, due to the

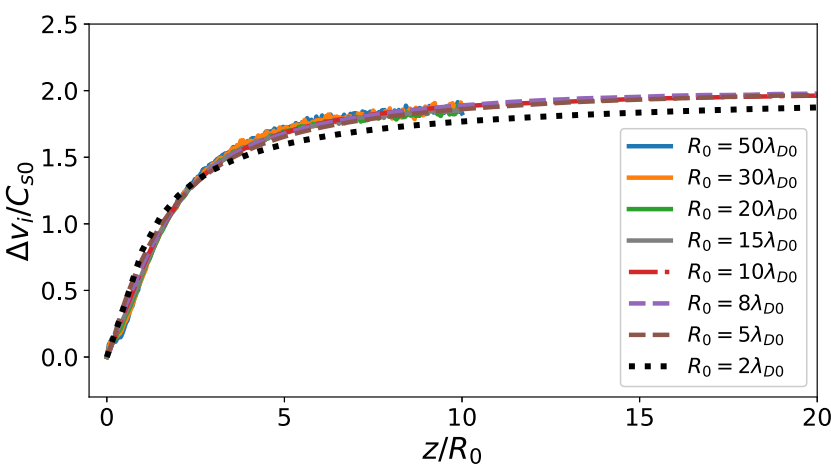

Figure 6. Ion velocity increase along the beam axis: comparison of all PIC cases.

small beam radius, the core beam region is perturbed by the effect of the expansion fan $[27,28]$. Figure 5 demonstrates that the expansion of a collisionless plasma beam is geometrically self-similar. The expansion characteristics is not affected by the source dimension if the source radius is sufficiently large, i.e. $R_{0}$ larger than a few $\lambda_{\mathrm{D} 0}$. The comparison reveals that the self-similarity is also shown in the data of experiments carried out by Corr et al [16], although it was not recognized.

We next examine the effect of source size on ion acceleration based on PIC simulation results. Figure 6 shows the ion velocity change along the beam axis. The velocity change is normalized by the ion acoustic speed at source exit, $\Delta v_{\mathrm{i}} / C_{s 0}=\left(v_{\mathrm{i}}-v_{0}\right) / C_{s 0}$. Again, to demonstrate the selfsimilarity, the axial position is normalized by the initial beam radius, $z / R_{0}$. Figure 6 shows that the $\Delta v(z)$ profiles are sizeindependent for $R_{0} \geqslant 5 \lambda_{\mathrm{D} 0}$, and that $\Delta v_{\mathrm{i}}$ for all different source sizes eventually reaches the same maximum value of approximately $2 C_{s 0}$. It is worth noting that the ion velocity reaches this maximum at $z / R_{0} \gtrsim 20$. This observation may have an important implication on laboratory testing of plasma sources. For instance, in order to avoid prematurely terminating plume expansion in laboratory testing, a vacuum chamber should have a length of at least $20 R_{0}$. In fact, the measured plasma density profile in [16] is observed to slightly rise near the end of the chamber wall for the $R_{0}=3 \mathrm{~cm}$ source (see also figure 5). This is probably due to a premature termination of beam expansion because the ratio of chamber length to the source radius $L_{\mathrm{c}} / R_{0}$ is only about 9 in this case.

\subsection{Analytical model}

We next derive an analytical model to estimate the ion acceleration. As the self-similarity is demonstrated, only the $R_{0}$ $=10 \lambda_{\mathrm{D} 0}$ case from the PIC result is used for comparison hereafter. For a collisionless ion flow, we can evaluate ion acceleration from conservation of energy along the ion streamline

$$
\begin{gathered}
\Delta v_{\mathrm{i}, \text { anal }}(z)=v_{\mathrm{i}, \text { anal }}(z)-v_{0}, \\
v_{\mathrm{i}, \text { anal }}(z)=\sqrt{v_{0}^{2}-2 e \Phi_{\text {anal }}(z) / m_{\mathrm{i}}},
\end{gathered}
$$

where the subscript 'anal' means the analytically computed quantities. To relate the plasma density change to the electric potential variation analytically, we assume that the electrons 


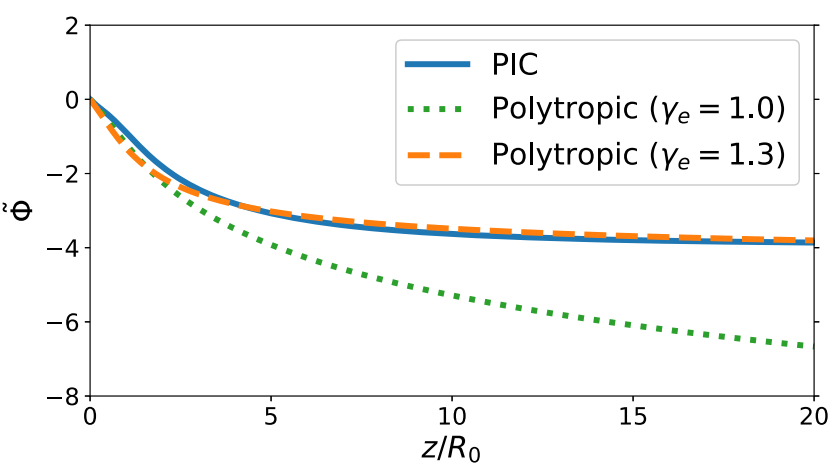

Figure 7. Electric potential along the beam axis: comparison of PIC result $\left(R_{0} / \lambda_{\mathrm{D} 0}=10\right)$ with analytical model.

can be modeled as a massless fluid and satisfy the polytropic relation,

$$
T_{\mathrm{e}} / T_{\mathrm{e} 0}=\left(n_{\mathrm{e}} / n_{\mathrm{e} 0}\right)^{\gamma_{\mathrm{e}}-1},
$$

where $\gamma_{\mathrm{e}}$ is the electron polytropic exponent determined by the thermodynamic process during expansion. Then, from quasineutrality, we have

$$
\tilde{\Phi}(z)= \begin{cases}\ln \left[\frac{n(z)}{n_{0}}\right] & \text { for } \gamma_{\mathrm{e}}=1 \\ \frac{\gamma_{\mathrm{e}}}{\gamma_{\mathrm{e}}-1}\left\{\left[\frac{n(z)}{n_{0}}\right]^{\gamma_{\mathrm{e}}-1}-1\right\} & \text { for } \gamma_{\mathrm{e}} \neq 1\end{cases}
$$

where $\tilde{\Phi}(z)=\frac{e \Phi(z)}{k_{\mathrm{b}} T_{\mathrm{c}}}$ is the normalized potential. Note that $\gamma_{\mathrm{e}}=1$ corresponds to the isothermal process, and yields the well-known Boltzmann relation. Though the Botlzmann relation is the most widely used model for plasma expansion problems [1-3,29-31], recent studies have shown that it overpredicts the potential drop in beam expansion [5, 20, 32]. This can be clearly seen in figure 7 , where the comparison shows that a polytropic coefficient $\gamma_{\mathrm{e}}=1.3$ matches with the PIC result. For the geometric expansions of plasmas with an inflow condition similar to the present setup, $[33,34]$ determined $\gamma_{\mathrm{e}}$ to be 1.2 , close to our value. Note that the isothermal assumption is unphysical because it implies that there exists an infinite amount of energy to drive the expansion [2, 3]. However, the exact cooling process for electrons in an expanding plasma is case-dependent.

Following the concept of virtual source from Burm et al [33], the axial density evolution in the expansion of plasma from a source can be given as

$$
\frac{n(z)}{n_{0}}=\frac{R_{0}^{2}}{R^{2}}=\frac{1}{1+z^{2} / z_{0}^{2}}
$$

where $z_{0}$ is the virtual source location. The virtual source concept is analogous to the spherical expansion of hydrodynamic flow from a source, where the density is inversely proportional to $R^{2}$. Such an assumption can be justified by the spherical shape of density contours shown in figure 4 . The choice of the virtual source location $z_{0}$ is important for the accuracy of the model, but is somewhat arbitrary in the literature. In references $[16,33], z_{0}$ is set to be the source radius $R_{0}$, corresponding to a $45^{\circ}$ expanding plasma. However, as shown in figure 4 , the

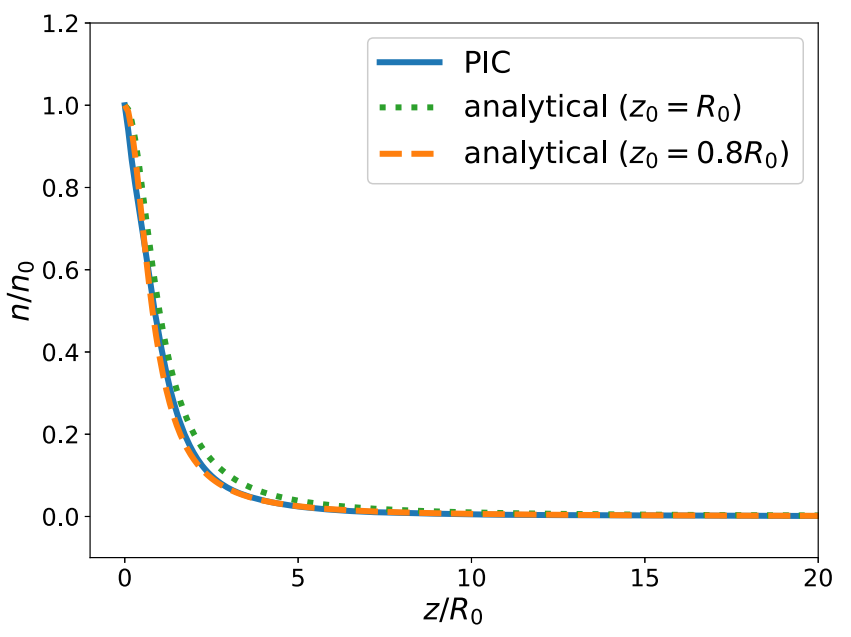

Figure 8. Ion number along the beam axis: comparison of PIC result $\left(R_{0} / \lambda_{\mathrm{D} 0}=10\right)$ with analytical model.

expansion angle of plasma plume is apparently larger than $45^{\circ}$. We find that the expansion angle is about $51^{\circ}$ and then the correct $z_{0}$ is $0.8 R_{0}$. Figure 8 compares the axial density profiles calculated by equation (5) and the PIC result. The analytical results for both $z_{0}=R_{0}$ and $0.8 R_{0}$ are presented for a comparison. We find that the model using $z_{0}=0.8 R_{0}$ shows an excellent agreement with PIC. Furthermore, the model with $z_{0}=0.8 R_{0}$ is observed to have a higher accuracy over $z_{0}=R_{0}$ in the entire region, demonstrating the validity of relating the virtual source location $z_{0}$ to the expansion angle.

The analytical model for the ion acceleration is obtained by integrating equations (4) and (5) into equation (3),

$$
\begin{aligned}
\Delta v_{\mathrm{i}, \text { anal }}(z) & =v_{\mathrm{i}, \text { anal }}(z)-v_{0}, \\
& =\sqrt{v_{0}^{2}-\frac{2 k_{\mathrm{b}} T_{0}}{m_{\mathrm{i}}} \frac{\gamma_{\mathrm{e}}}{\gamma_{\mathrm{e}}-1}\left\{\left[\frac{1}{1+\left(1.25 z / R_{0}\right)^{2}}\right]^{\gamma_{\mathrm{e}}-1}-1\right\}}-v_{0},
\end{aligned}
$$

where $\gamma_{\mathrm{e}}=1.3$ is used. Figure 9 compares the analytical solution of equation (6) with the PIC result. The analytical solution agrees very well with the PIC result. The maximum relative error $\left|v_{\mathrm{i}, \text { anal }}-v_{\mathrm{i} \text {,PIC }}\right| / v_{\mathrm{i} \text {,PIC }}$ is about $6 \%$, and occurs at $z \approx R_{0}$. For the saturation ion speed, the relative error of analytical solution with respect to the PIC result is about $1 \%$.

\subsection{Effects from electron cooling}

The results in previous sections suggest that the electrons during the expansion may be approximated by a gas dynamic-like cooling process with a polytropic coefficient $\gamma_{\mathrm{e}} \simeq 1$.3. However, the electron cooling in a collisionless plasma is not a trivial process. This section further examines electron cooling during expansion.

If the electrons are initially Maxwellian with temperature $T_{0}$ and only the electrostatic field is involved, the Vlasov equation predicts that the electron density scales with $\exp \left(-e \Phi / k_{\mathrm{b}} T_{0}\right)$ while the Maxwellian distribution is maintained $[35,36]$. The electron temperature is therefore constant 


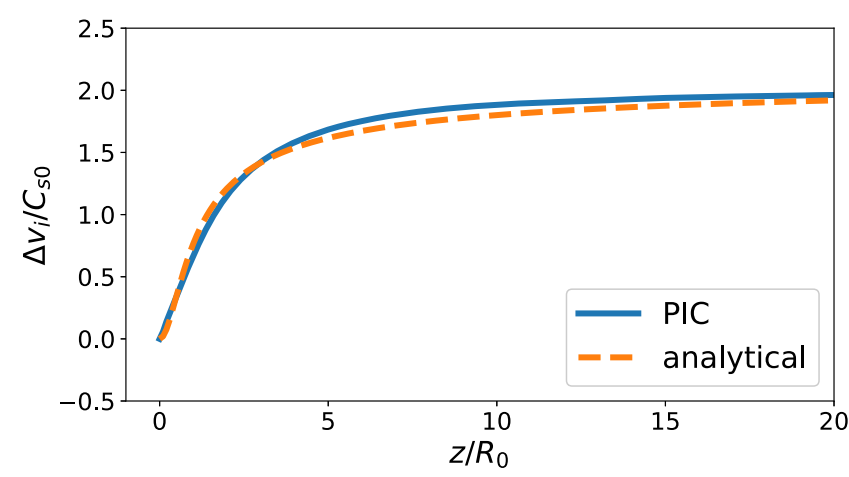

Figure 9. Ion velocity increase along the beam axis: comparison of PIC result $\left(R_{0} / \lambda_{\mathrm{D} 0}=10\right)$ with analytical model.

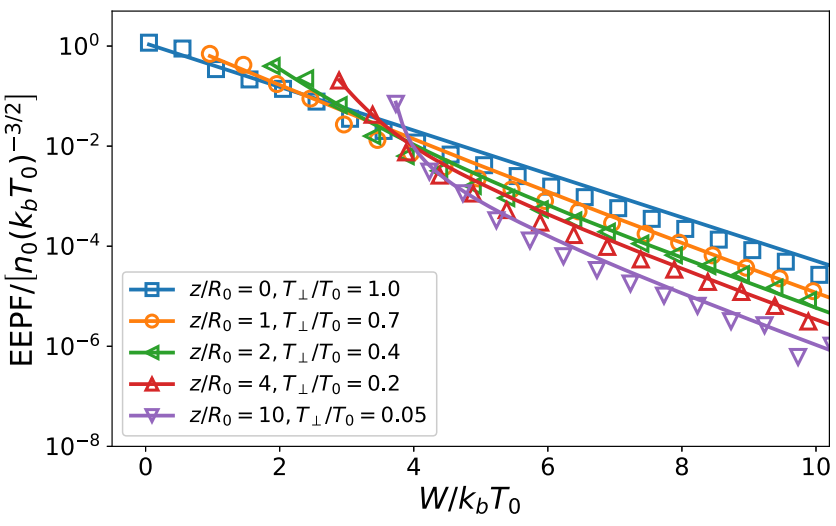

Figure 10. Local EEPF at selected locations along the beam axis. The PIC results are shown by the symbols. The solid lines are the fitting curves using the bi-Maxwellian function.

in this situation, as demonstrated in reference [37]. A polytropic coefficient of $\gamma_{\mathrm{e}} \simeq 1.3$ in this study suggests that the electrons deviate from equilibrium during the expansion.

Consider the electron energy probability function (EEPF) for Maxwellian electrons

$$
g_{\mathrm{M}}(\varepsilon, \mathbf{x})=n \frac{2}{\sqrt{\pi}} \frac{1}{\left(k_{\mathrm{b}} T\right)^{3 / 2}} \exp \left(-\frac{\varepsilon}{k_{\mathrm{b}} T}\right),
$$

where $\varepsilon$ indicates the kinetic energy of an electron, $n=n(\mathbf{x})$ and $T=T(\mathbf{x})$ are the local electron number density and temperature, respectively. Figure 10 shows the EEPF at selected positions along the beam center line. Here, EEPF is normalized by $n_{0}\left(k_{\mathrm{b}} T_{0}\right)^{-3 / 2}$ and plotted against the electron total energy $W=\varepsilon-e \Phi(\mathbf{x})$. On a semi-log plot, a Maxwellian EEPF is shown as a straight line whose slope is the temperature. It is evident from figure 10 that the electrons gradually deviate from Maxwellian as they expand into the downstream. The local EEFP starts to exhibit a concave shape as the electrons leave the source. Moreover, the slopes at different positions are almost identical for large $W$, i.e. $W \gtrsim 5 k_{\mathrm{b}} T_{0}$. The deviation of EEPF from the Maxwellian suggests that some electrons may be scattered into the loss cone in the phase-space during the expansion process [38]. This will need to be investigated further.
The concave-shaped EEPF suggests that the electrons can be represented by an anisotropic bi-Maxwellian type distribution. Indeed, previous studies have shown that the expansion of a collision plasma into vacuum can yield strong anisotropic electrons with an effective temperature along the beam direction, $T_{\|}$, larger than that in the radial or transverse direction, $T_{\perp}$ $[5,6,21]$. The EEPF for bi-Maxwellian electrons at position $\mathbf{x}$ reads

$$
\begin{aligned}
g_{\mathrm{BM}}(\varepsilon, \mathbf{x})= & n \frac{1}{k_{\mathrm{b}} T_{\perp}} \frac{1}{\left(k_{\mathrm{b}} T_{\|}\right)^{1 / 2}}\left(\frac{\varepsilon}{k_{\mathrm{b}} T_{\perp}}-\frac{\varepsilon}{k_{\mathrm{b}} T_{\|}}\right)^{-1 / 2} \\
& \times \operatorname{erfi}\left[\left(\frac{\varepsilon}{k_{\mathrm{b}} T_{\perp}}-\frac{\varepsilon}{k_{\mathrm{b}} T_{\|}}\right)^{1 / 2}\right] \exp \left(-\frac{\varepsilon}{k_{\mathrm{b}} T_{\perp}}\right),
\end{aligned}
$$

for $T_{\|}>T_{\perp}$, where $T_{\|}=T_{\|}(\mathbf{x})$ and $T_{\perp}=T_{\perp}(\mathbf{x})$ denote the local electron 'effective' temperature parallel and perpendicular to the beam velocity, respectively. As shown in figure 10, the EEPFs from PIC can well be fitted to the bi-Maxwellian form given by equation (8) if we keep $T_{\|}=T_{0}$ but allow a position-dependent $T_{\perp}$. Hence, the effect of electron cooling is mainly contributed from $T_{\perp}$. It is difficult to identify the exact mechanism responsible for the decrease of $T_{\perp}$ in a simple way because the nonlinear interaction between the electrons and electric field occurs in two-dimension. However, we can capture the dominant mechanism by analyzing the control volume along the beam center line, where there is no electric field in the radial direction due to symmetry. After the initially Maxwellian electrons enter the expansion region, the particles with a higher radial velocity leave the control volume faster radially. Consequently, the radial component of electron kinetic energy in the control volume keeps decreasing as the electrons expand downstream. In the axial direction, however, the electric field will eventually attract the electrons which escape the control volume back. In this situation, the Vlasov solution predicts that the electrons should follow the Boltzmann relation with its initial temperature $[35,36]$. Therefore, we conclude that the electron cooling is primarily due to the loss of electrons with high energies in the transverse direction.

\subsection{Effects from collisions}

For plasma sources used for spacecraft charging control, EP, and laboratory simulations of space plasmas, the beams emitted are typically near collisionless. However, it is still worthwhile to examine whether the self-similar may be affected by the existence of infrequent collisions.

Collisions have a larger impact on plasma beams in the vacuum chamber environment than under the space environment due to background gas and other facility effects [12]. Hence, we use the experiments in [16] as an example. The vacuum chamber in [16] was $28 \mathrm{~cm}$ long and $7 \mathrm{~cm}$ in radius, and the radii of the plasma sources were $1,2.25$, and $3 \mathrm{~cm}$. The typical operating condition produced a plasma with $n_{\mathrm{i}} \simeq n_{\mathrm{e}} \simeq 10^{18} \mathrm{~m}^{-3}$, and $T_{\mathrm{e}} \simeq 8 \mathrm{eV}$ at the source exit, and a chamber background pressure of $\simeq 0.6$ mTorr. The background pressure yields a neutral density of $n^{\text {neu }} \simeq 10^{19} \mathrm{~m}^{-3}$. 
The mean free paths of Coulomb and charge-exchange (CEX) collisions for the ions are estimated to be $\simeq 1 \mathrm{~m}$ and $\simeq 0.1 \mathrm{~m}$. Therefore, the Coulomb collision can be completely ignored as its mean free path is much larger than the chamber dimension. Only the effect of CEX collision needs to be evaluated.

We include a Monte Carlo collision (MCC) model, which simulates the collisions between beam ions with a background neutral gas, into the PIC model discussed in section 2 . We apply an analytical free molecular solution for the spatial distribution of neutrals $[39,40]$ to calculate the neutral gas background in the vacuum chamber. The density and velocity of the background neutrals are given as:

$$
\begin{gathered}
\frac{n^{\text {neu }}(z, r)}{n_{0}^{\text {neu }}}=\frac{\exp \left(-S_{0}^{2}\right)}{\sqrt{\pi^{3}} z^{2}} \int_{-\pi / 2}^{\pi / 2} \mathrm{~d} \theta \int_{0}^{R_{0}} r^{\prime} K \mathrm{~d} r^{\prime} \\
\frac{V_{z}^{\text {neu }}(z, r)}{\sqrt{2 k_{\mathrm{b}} T_{0}^{\text {neu }} / m^{\text {neu }}}}=\frac{n_{0}^{\text {neu }}}{n^{\text {neu }}} \frac{\exp \left(-S_{0}^{2}\right)}{\sqrt{\pi^{3}} z^{2}} \int_{-\pi / 2}^{\pi / 2} \mathrm{~d} \theta \int_{0}^{R_{0}} r^{\prime} M \mathrm{~d} r^{\prime}, \\
\frac{V_{\mathrm{r}}^{\text {neu }}(z, r)}{\sqrt{2 k_{\mathrm{b}} T_{0}^{\text {neu }} / m^{\text {neu }}}}=\frac{n_{0}^{\text {neu }}}{n^{\text {neu }}} \frac{\exp \left(-S_{0}^{2}\right)}{\sqrt{\pi^{3}} z^{2}} \int_{-\pi / 2}^{\pi / 2} \mathrm{~d} \theta \\
\times \int_{0}^{R_{0}}\left(r-r^{\prime} \sin \theta\right) r^{\prime} M \mathrm{~d} r^{\prime},
\end{gathered}
$$

with the following integrand factors:

$$
\begin{aligned}
K= & Q\left\{Q S_{0}+\left(\frac{1}{2}+Q S_{0}^{2}\right) \sqrt{Q \pi}\right. \\
& \left.\times\left[1+\operatorname{erf}\left(\sqrt{Q} S_{0}\right) e^{Q S_{0}^{2}}\right]\right\}, \\
M= & Q^{2}\left\{Q S_{0}^{2}+1+S_{0}\left(\frac{3}{2}+Q S_{0}^{2}\right) \sqrt{Q \pi}\right. \\
\times & {\left.\left[1+\operatorname{erf}\left(\sqrt{Q} S_{0}\right) e^{Q S_{0}^{2}}\right]\right\}, } \\
Q= & \frac{z^{2}}{z^{2}+r^{2}+r^{\prime 2}-2 r r^{\prime} \sin \theta} .
\end{aligned}
$$

In equations (9)-(11), the integration is over the source exit surface; the superscript 'neu' represents the neutral properties while the subscript ' 0 ' denotes the source condition; $S_{0}=V_{0}^{\text {neu }} / \sqrt{2 k_{\mathrm{b}} T_{0}^{\text {neu }} / m^{\text {neu }}}$ is the speed ratio.

Equations (9)-(11) are integrated numerically to produce the flow characteristics for the neutral background, and then used as input in our PIC-MCC model. At each simulation step, the number of CEX ions in cell $(i z, i r)$ are produced according to

$$
\Delta N_{\text {cex }}(i z, i r)=\left(n^{\text {neu }} n_{\mathrm{i}} v_{\mathrm{i}} V\right)_{(i z, i r)} \sigma_{\text {cex }} \Delta t,
$$

where $n_{\mathrm{i}}$ and $v_{\mathrm{i}}$ are the beam ion density and velocity, $V$ is the volume of cell $(i r, i r), \sigma_{\text {cex }}$ is CEX collision cross section. The initial positions of generated CEX ions are randomly distributed in the cell, and the initial velocity is assigned according to equations (10) and (11).
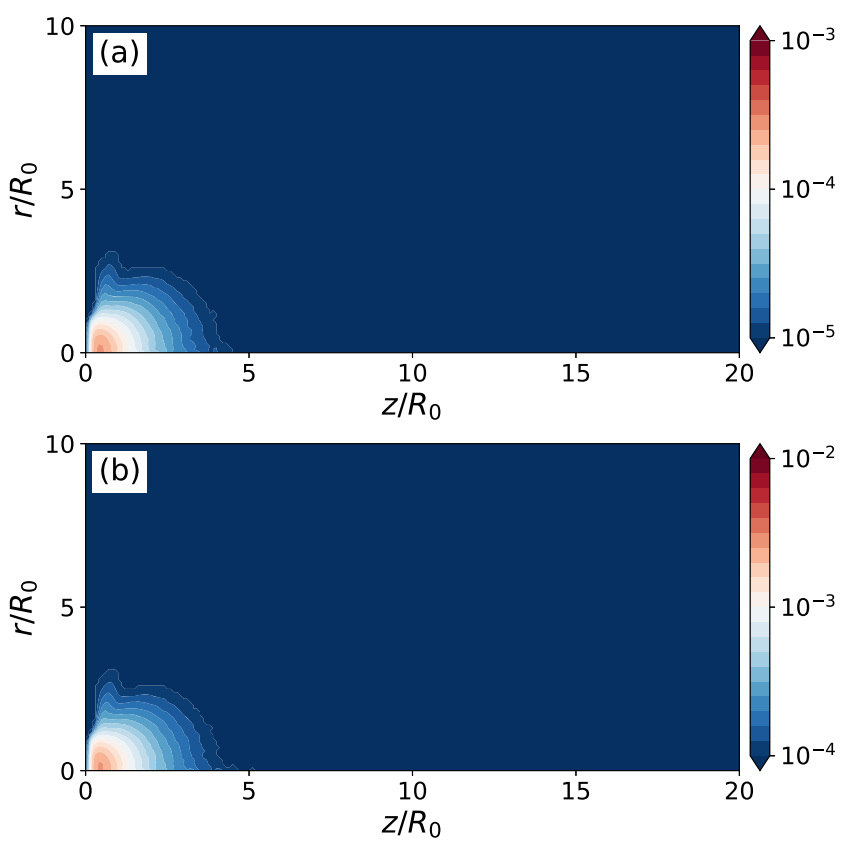

Figure 11. CEX ion number density contour. (a) $n_{0}^{\text {neu }} / n_{0}=10$. (b) $n_{0}^{\text {neu }} / n_{0}=100$.

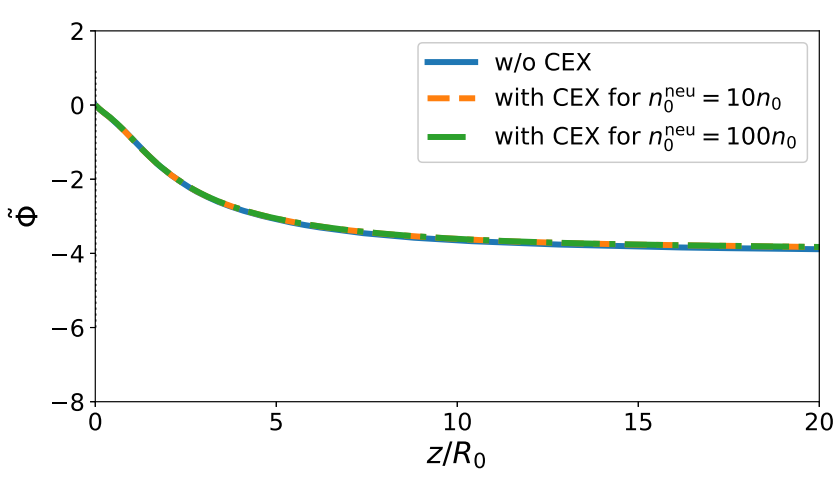

Figure 12. Electric potential along the beam axis: effects from CEX collisions.

We carried out PIC-MCC simulations with $T_{0}^{\text {neu }}=$ $500 \mathrm{~K}, S_{0}=0$, and $n_{0}^{\text {neu }}=10^{19}$ and $10^{20} \mathrm{~m}^{-3}$ (or equivalently $n_{0}^{\text {neu }} / n_{0}=10$ and 100) to investigate effect of CEX. In [16], the beam ion energy is approximately a few eV. According to [41], $\sigma_{\text {cex }}$ is considered to be constant $5 \times 10^{-19} \mathrm{~m}^{2}$ for simplicity in this study.

Figure 11 shows the distribution of CEX ions normalized by $n_{0}$. It is evident that the CEX ions exist only in a limited region close to the source exit, where both the neutral and beam ion densities are high. However, the number density of the CEX ions is still several orders of magnitude lower than that of the beam ions even if we assume $n_{0}^{\text {neu }} / n_{0}=100$, a value much higher than that in the actual experiment. Due to the low CEX ion density, the effect of the CEX ions on beam expansion and, thereby, the self-similarity, is negligible. This is demonstrated by the potential profile in figure 12. As expected, for collisionless or near-collisionless plasma expansion, the self-similarity is not affected by infrequent collisions in the beam. 


\section{Conclusions}

This paper investigates the self-similarity in the collisionless plasma beam expansion. For most applications related to EP, spacecraft charging control, and laboratory simulations of space plasmas, the beams emitted from a source are in the collisionless regime. Axi-symmetric fully kinetic PIC simulations and analytical studies are carried out to investigate the expansion of a cylindrical plasma beam into a vacuum with $M=v_{0} / C_{s}=1$ at the emission plane. PIC simulation results agree well with the available experiments carried out at similar conditions. We find that the ion dynamics during expansion is geometrically self-similar if the initial beam radius is sufficiently large $\left(R_{0} \geqslant 5 \lambda_{\mathrm{D} 0}\right.$ for the case considered). The results show that the beam ions are accelerated to a maximum speed approximately equal to $v_{0}+2 C_{s 0}$ at $z \approx 20 R_{0}$ downstream of the source exit. This implies that, for laboratory testing of plasma sources, the vacuum chamber facility may significantly affect the plasma beam by prematurely terminating the expansion if the chamber wall is less than $20 R_{0}$ downstream of the plasma source exit. Fully kinetic PIC simulations also show that the effective polytropic exponent for the electrons in the beam is $\simeq 1.3$. Using a virtual source model for plasma beam density evolution and the polytropic model for the electrons derived from the full PIC simulations, an analytical method capable of accurately predicting beam ion acceleration is proposed. The results obtained in this study can be used as a benchmark to measure the performance of those plasma sources emitting a beam at $M \simeq 1$, such as magnetic nozzle devices. Ion accelerations in expanding plasma beams emitted at other Mach numbers will be addressed in a future study.

\section{Acknowledgments}

YH was partially supported by the LHD Youth Innovation Fund from the State Key Laboratory of High Temperature Gas Dynamics (Grant No. LHD2019CX12). QS was supported by the Strategic Priority Research Program of the Chinese Academy of Sciences (Grant No. XDA17030100). YH would also like to thank Dr. Min Li from the Innovation Academy for Microsatellites of the Chinese Academy of Sciences for the helpful discussion on the details of particle boundary condition.

\section{ORCID iDs}

Yuan $\mathrm{Hu}$ (D) https://orcid.org/0000-0001-6870-183X

Joseph Wang (D) https://orcid.org/0000-0001-8032-4352

\section{References}

[1] Gurevich A V, Pariiskaya L V and Pitaevskii L P 1966 Sov. Phys. JETP 22 449-54
[2] Crow J E, Auer P L and Allen J E 1975 J. Plasma Phys. 14 $65-76$

[3] Mora P 2003 Phys. Rev. Lett. 90185002

[4] Arefiev A V and Breizman B N 2009 Phys. Plasmas 16055707

[5] Hu Y and Wang J 2018 Phys. Rev. E 98023204

[6] Wang J and Hu Y 2019 Phys. Plasmas 26103502

[7] Li M, Merino M, Ahedo E and Tang H 2019 Plasma Sources Sci. Technol. 28034004

[8] Correyero S, Jarrige J, Packan D and Ahedo E 2019 Plasma Sources Sci. Technol. 28095004

[9] Alexander B, Charles C and Boswell R 2018 Plasma Sources Sci. Technol. 27075003

[10] Bennet A, Charles C and Boswell R W 2019 Plasma Sources Sci. Technol. 28 06LT01

[11] Polansky J, Wang J and Ding N 2013 IEEE Trans. Plasma Sci. $413438-47$

[12] Wang J, Han D and Hu Y 2015 IEEE Trans. Plasma Sci. 43 3047-53

[13] Zhang Z, Ren J, Tang H, Cao J, Cao S, Chen Z, Ling W Y L and Zhao R 2018 Vacuum 155 199-209

[14] Taccogna F, Longo S, Capitelli M and Schneider R 2005 Phys. Plasmas 12053502

[15] Yuan T, Ren J, Zhou J, Zhang Z, Wang Y and Tang H 2020 AIP $A d v .10045115$

[16] Corr C S, Zanger J, Boswell R W and Charles C 2007 Appl. Phys. Lett. 91241501

[17] Corr C S, Boswell R W, Charles C and Zanger J 2008 Appl. Phys. Lett. 92221508

[18] Denavit J 1979 Phys. Fluids 22 1384-92

[19] Wang J, Chang O and Cao Y 2012 IEEE Trans. Plasma Sci. 40 230-6

[20] Hu Y and Wang J 2015 IEEE Trans. Plasma Sci. 43 2832-8

[21] Hu Y and Wang J 2017 Phys. Plasmas 24033510

[22] Zhao Y, Wang J and Usui H 2018 J. Propul. Power 34 1109-15

[23] Brieda L 2018 IEEE Trans. Plasma Sci. 46 556-62

[24] Birdsall C K and Langdon A B 2004 Plasma Physics via Computer Simulation (Boca Raton, FL: CRC Press)

[25] Othmer C, Glassmeier K H, Motschmann U, Schüle J and Frick C 2000 Phys. Plasmas 7 5242-51

[26] Singh N, Rao S and Ranganath P 2013 Phys. Plasmas 2003211

[27] Wang J and Hastings D E 1992 Phys. Fluids B 4 1597-614

[28] Wang J and Hastings D E 1992 Phys. Fluids B 4 1615-29

[29] Allen J E and Andrews J G 1970 J. Plasma Phys. 4 187-94

[30] Perego M, Howell P D, Gunzburger M D, Ockendon J R and Allen J E 2013 Phys. Plasmas 20052101

[31] Allen J E and Perego M 2014 Phys. Plasmas 21034504

[32] Hu Y and Wang J 2019 IEEE Trans. Plasma Sci. 47 3731-8

[33] Burm K T A L, Goedheer W J and Schram D C 2001 J. Appl. Phys. 90 2162-8

[34] Burm K T A L, Jodoin B, Proulx P and Boulos M I 2003 Plasma Sources Sci. Technol. 12 362-71

[35] Krall N A and Trivelpiece A W 1973 Principles of Plasma Physics (New York: McGraw-Hill)

[36] Gurnett D A and Bhattacharjee A 2017 Introduction to Plasma Physics (With Space and Laboratory Applications) (Cambridge: Cambridge University Press)

[37] Boswell R, Takahashi K, Charles C and Kaganovich I 2015 Front. Phys. 3 1-5

[38] Kaganovich I D et al 2020 Perspectives on physics of $E \times B$ discharges relevant to plasma propulsion and similar technologies (arXiv:2007.09194)

[39] Cai C and Huang X 2012 AIAA J. 50 2908-18

[40] Cai S, Cai C and Li J 2019 Phys. Plasmas 26052109

[41] Vahedi V and Surendra M 1995 Comput. Phys. Commun. 87 179-98 